\title{
Triple Benzylic Dehydrogenation by Osmium in an Amide Ligand
}

\author{
Environment \\ Joo-Ho Lee, Maren Pink, and Kenneth G. Caulton* \\ Department of Chemistry, Indiana University, Bloomington, IN
}

\section{Supporting Information}

General Considerations. All manipulations were performed using standard Schlenk techniques or in an argon filled glovebox unless otherwise noted. Solvents were distilled from $\mathrm{Na}, \mathrm{Na}$ /benzophenone, or $\mathrm{CaH}_{2}$, degassed prior to use, and stored over $4 \AA$ molecular sieves in air-tight vessels. All NMR solvents were also dried, vacuum transferred and stored in the glovebox under argon. All other reagents were used as received from commercial vendors. $[\mathrm{LiPNP}]_{2} \mathrm{LiCl}$ was synthesized according to a published procedure ${ }^{1}$ using $\mathrm{HP}^{\mathrm{t}} \mathrm{Bu}_{2}$ instead of $\mathrm{HP}^{\mathrm{i}} \mathrm{Pr}_{2}$. [CymeneOsCl$\left.]_{2}\right]_{2}$ was synthesized according to a published procedure ${ }^{2}$. NMR chemical shifts are reported in ppm relative to protio impurities in the deutero solvents. ${ }^{31} \mathrm{P}$ NMR or ${ }^{13} \mathrm{C}$ NMR spectra are referenced to external standards of $\mathrm{H}_{3} \mathrm{PO}_{4}$ or natural abundance ${ }^{13} \mathrm{C}$ peak of the solvent respectively. NMR spectra were recorded with a Varian Gemini $2000\left(300 \mathrm{MHz}{ }^{1} \mathrm{H} ; 121 \mathrm{MHz}^{31} \mathrm{P} ; 75\right.$ $\left.\mathrm{MHz}{ }^{13} \mathrm{C}\right)$ or a Varian Unity INOVA instrument $\left(400 \mathrm{MHz}{ }^{1} \mathrm{H} ; 162 \mathrm{MHz}{ }^{31} \mathrm{P} ; 101 \mathrm{MHz}\right.$ ${ }^{13} \mathrm{C}$ ). Infrared spectra were recoded on a Nicolet 510P FT-IR spectrometer. Below “Ar" is $\mathrm{C}_{6} \mathrm{H}_{4}{ }^{\mathrm{i}} \mathrm{Pr}$.

\section{$(\mathrm{PNP}) \mathrm{Os}(\mathrm{H})_{2}(\mathrm{CAr})$}

$10 \mathrm{mg}$ of $[\mathrm{LiPNP}]_{2} \mathrm{LiCl}(10 \mu \mathrm{mol})$ was added into the solution of $4 \mathrm{mg}$ of [CymeneOsCl $]_{2}(5 \mu \mathrm{mol})$ (in $0.5 \mathrm{~mL}$ of benzene) in a J-Young tube. After 24 hours, 5 $\mu \mathrm{L}$ of MeI was added into the reaction mixture and then stirred 4 hours more. The volatiles were then removed in vacuo. Pentane was added to the reaction tube, the solution was filtered and the filtrate was dried in vacuo. NMR spectra indicated complete

\footnotetext{
${ }_{1}^{1}$ Fryzuk. M. D., Giesbrecht. G. R., Tettig, S. J. Organometallics 1997, 16, 725.

${ }^{2}$ Werner, H., Zenkert, K. J. Organomet. Chem. 1988, 345, 151.
} 
conversion to (PNP)Os( $\mathrm{H})_{2}(\mathrm{CAr})$ from $[\mathrm{CymeneOsCl}]_{2} .{ }^{1} \mathrm{H} \mathrm{NMR}\left(\mathrm{C}_{6} \mathrm{D}_{6}, 25{ }^{\circ} \mathrm{C}\right):-1.02$ $\operatorname{ppm}\left(\mathrm{t}, \mathrm{J}_{\mathrm{PH}}=14.7 \mathrm{~Hz}, 2 \mathrm{H}, \mathrm{OsH}_{2}\right), 0.42(\mathrm{~s}, 12 \mathrm{H}, \mathrm{SiMe}), 0.92\left(\mathrm{~d}, \mathrm{~J}_{\mathrm{HH}}=6.9 \mathrm{~Hz}, 6 \mathrm{H}\right.$, $\mathrm{CHMe}_{2}$ ), 1.28 (vt, J = 6.9 Hz, 4H, $\left.\mathrm{SiCH}_{2}\right), 1.36$ (vt, J = 6.3 Hz, 36H, P $\left.{ }^{t} \mathrm{Bu}_{2}\right), 2.41(\mathrm{~m}, 1 \mathrm{H}$, $\left.\mathrm{CHMe}_{2}\right), 6.79\left(\mathrm{~d}, \mathrm{~J}_{\mathrm{HH}}=7.8 \mathrm{~Hz}, 2 \mathrm{H}, \mathrm{Ar}\right), 7.57\left(\mathrm{~d}, \mathrm{~J}_{\mathrm{HH}}=7.8 \mathrm{~Hz}, 2 \mathrm{H}, A r\right) .{ }^{31} \mathrm{P}\left\{{ }^{1} \mathrm{H}\right\} \mathrm{NMR}$ $\left(\mathrm{C}_{6} \mathrm{D}_{6}, 25{ }^{\circ} \mathrm{C}\right): 58.4 \mathrm{ppm}(\mathrm{s}) .{ }^{13} \mathrm{C}\left\{{ }^{1} \mathrm{H}\right\} \operatorname{NMR}\left(\mathrm{C}_{6} \mathrm{D}_{6}, 25{ }^{\circ} \mathrm{C}\right): 259 \mathrm{ppm}\left(\mathrm{t}, \mathrm{J}_{\mathrm{CP}}=11 \mathrm{~Hz}\right.$, $\mathrm{Os} C)$.

\section{(PNP)Os(H) 3}

$(\mathrm{PNP}) \mathrm{Os}(\mathrm{H})_{2}(\mathrm{CAr})(10 \mu \mathrm{mol})$ in a J-Young tube was dissolved in $0.5 \mathrm{~mL}$ of $\mathrm{C}_{6} \mathrm{H}_{6}$ and was degassed through 3 freeze-pump-thaw cycles using liquid $\mathrm{N}_{2}$. 1 atm of $\mathrm{H}_{2}$ was added to the evacuated head space of the frozen solution in the tube and the solution was thawed and agitated. After 10 minutes, 1 atm of $\mathrm{H}_{2}$ was added again. After 1 hour, the volatiles were removed in vacuo. By NMR spectra, complete conversion to $(\mathrm{PNP}) \mathrm{Os}(\mathrm{H})_{3}$ from (PNP)Os $(\mathrm{H})_{2}(\mathrm{CAr})$ was observed. ${ }^{1} \mathrm{H} \mathrm{NMR}\left(\mathrm{C}_{6} \mathrm{D}_{6}, 25{ }^{\circ} \mathrm{C}\right):-16.56 \mathrm{ppm}\left(\mathrm{t}, \mathrm{J}_{\mathrm{PH}}=\right.$ $11.1 \mathrm{~Hz}, 3 \mathrm{H}, \mathrm{OsH}_{3}$ ), 0.41 (s, 12H, SiMe $), 0.94$ (vt, J = 4.5Hz, 4H, $\mathrm{SiCH}_{2}$ ), 1.19 (vt, J = $\left.6 \mathrm{~Hz}, 36 \mathrm{H}, \mathrm{P}^{t} \mathrm{Bu}_{2}\right) .{ }^{31} \mathrm{P}\left\{{ }^{1} \mathrm{H}\right\} \mathrm{NMR}\left(\mathrm{C}_{6} \mathrm{D}_{6}, 25{ }^{\circ} \mathrm{C}\right): 69.8 \mathrm{ppm}(\mathrm{s})$.

\section{(PNP)OsH(CO)(CHAr)}

$(\mathrm{PNP}) \mathrm{Os}(\mathrm{H})_{2}(\mathrm{CAr})(10 \mu \mathrm{mol})$ in a J-Young tube was dissolved in $0.5 \mathrm{~mL}$ of $\mathrm{C}_{6} \mathrm{D}_{6}$ and was degassed through 3 freeze-pump-thaw cycles using liquid $\mathrm{N}_{2} .1$ atm of $\mathrm{CO}$ was added to the evacuated head space of the frozen solution in the tube and the solution was melted and shaken. Immediate color changed was observed from reddish brown to green. After 30 minutes, the volatiles were removed in vacuo. By NMR spectra, complete conversion to (PNP)OsH(CO)(CHAr) was observed. ${ }^{1} \mathrm{H}$ NMR $\left(\mathrm{C}_{6} \mathrm{D}_{6}, 25{ }^{\circ} \mathrm{C}\right):-2.72 \mathrm{ppm}$ $\left(\mathrm{dt}, \mathrm{J}_{\mathrm{HH}}=6.3 \mathrm{~Hz}, \mathrm{~J}_{\mathrm{HP}}=21 \mathrm{~Hz}, \mathrm{H}, \mathrm{OsH}\right), 0.48(\mathrm{~s}, 6 \mathrm{H}, \mathrm{SiMe}), 0.59(\mathrm{~s}, 6 \mathrm{H}, \mathrm{SiMe}), 1.00(\mathrm{~d}$, $\left.\mathrm{J}_{\mathrm{HH}}=6.6 \mathrm{~Hz}, 6 \mathrm{H}, \mathrm{CHMe} e_{2}\right), 1.10\left(\mathrm{vt}, \mathrm{J}=6.3 \mathrm{~Hz}, 18 \mathrm{H}, \mathrm{P}^{t} \mathrm{Bu}\right), 1.22(\mathrm{vt}, \mathrm{J}=6.3 \mathrm{~Hz}, 18 \mathrm{H}$, $\left.\mathrm{P}^{t} \mathrm{Bu}\right), 2.43\left(\mathrm{sep}, \mathrm{J}_{\mathrm{HH}}=6.6 \mathrm{~Hz} 1 \mathrm{H}, \mathrm{CHMe}_{2}\right), 7.01$ (s, 2H, Ar), 7.47 (bs, 1H, Ar), 9.15 (bs, $1 \mathrm{H}, A r), 19.47\left(\mathrm{dt}, \mathrm{J}_{\mathrm{HH}}=6.3 \mathrm{~Hz}, \mathrm{~J}_{\mathrm{HP}}=5.6 \mathrm{~Hz}, 1 \mathrm{H}, \mathrm{CHAr}\right) .{ }^{31} \mathrm{P}\left\{{ }^{1} \mathrm{H}\right\} \mathrm{NMR}\left(\mathrm{C}_{6} \mathrm{D}_{6}, 25{ }^{\circ} \mathrm{C}\right)$ : 53.0 ppm (s). ${ }^{13} \mathrm{C}\left\{{ }^{1} \mathrm{H}\right\} \operatorname{NMR}\left(\mathrm{C}_{6} \mathrm{D}_{6}, 25{ }^{\circ} \mathrm{C}\right)$ : $269.6 \mathrm{ppm}$ (bs, OsC), 194.9 (bs, Os $\left.(\mathrm{CO})\right)$. 


\section{Isomerization from (PNP)OsH(CO)(CHAr) to (PNP)Os(CO)( $\left.\mathrm{CH}_{2} \mathrm{Ar}\right)$}

(PNP)OsH(CO)(CHAr) $(10 \mu \mathrm{mol})$ in a J-Young tube was placed into a $65^{\circ} \mathrm{C}$ bath for 4 hours. A color change was observed from green to brown. By NMR spectra, complete isomerization to (PNP)Os( $\mathrm{CO})\left(\mathrm{CH}_{2} \mathrm{Ar}\right)$ was established. ${ }^{1} \mathrm{H}$ NMR $\left(\mathrm{C}_{6} \mathrm{D}_{6}, 25\right.$ ${ }^{\circ} \mathrm{C}$ ): $0.40 \mathrm{ppm}$ (s, 6H, SiMe), 0.47 (s, 6H, SiMe), 1.12 (vt, J = 6.3 Hz, 18H, P ${ }^{t} B u$ ), 1.19 (vt, $\mathrm{J}=6.3 \mathrm{~Hz}, 18 \mathrm{H}, \mathrm{P}^{t} \mathrm{Bu}$ ), $2.74\left(\mathrm{sep}, \mathrm{J}_{\mathrm{HH}}=6.9 \mathrm{~Hz} 1 \mathrm{H}, \mathrm{CHMe}\right), 4.76\left(\mathrm{t}, \mathrm{J}_{\mathrm{HP}}=4.4 \mathrm{~Hz}, 2 \mathrm{H}\right.$, $\left.\mathrm{CH}_{2} \mathrm{Ar}\right), 7.05\left(\mathrm{~d}, \mathrm{~J}_{\mathrm{HH}}=8.1 \mathrm{~Hz}, 2 \mathrm{H}, \mathrm{Ar}\right), 7.66\left(\mathrm{~d}, \mathrm{~J}_{\mathrm{HH}}=8.1 \mathrm{~Hz}, 2 \mathrm{H}, A r\right) .{ }^{31} \mathrm{P}\left\{{ }^{1} \mathrm{H}\right\} \mathrm{NMR}$ $\left(\mathrm{C}_{6} \mathrm{D}_{6}, 25^{\circ} \mathrm{C}\right): 38.8 \mathrm{ppm}$ (s). IR (pentane solution): $v(\mathrm{C} \equiv \mathrm{O}) 1865 \mathrm{~cm}^{-1}$.

\section{(PNP)OsH(CO)}

(PNP)Os(CO) $\left(\mathrm{CH}_{2} \mathrm{Ar}\right)(10 \mu \mathrm{mol})$ in a J-Young tube was dissolved in $0.5 \mathrm{~mL}$ of $\mathrm{C}_{6} \mathrm{D}_{6}$ and was degassed through 3 freeze-pump-thaw cycles using liquid $\mathrm{N}_{2} .1 \mathrm{~atm}$ of $\mathrm{H}_{2}$ was added to the evacuated head space of the frozen solution in the tube and the solution was melted and shaken. Immediate color changed was observed from brown to pale yellow. After 30 minutes, the volatiles were removed in vacuo. ${ }^{1} \mathrm{H} N M R\left(\mathrm{C}_{6} \mathrm{D}_{6}, 25^{\circ} \mathrm{C}\right)$ : $-29.46 \mathrm{ppm}\left(\mathrm{t}, \mathrm{J}_{\mathrm{PH}}=13.2 \mathrm{~Hz}, 1 \mathrm{H}, \mathrm{OsH}\right.$ ), 0.32 (s, 6H, SiMe), 0.33 (s, 6H, SiMe), 1.23 (vt, $\left.\mathrm{J}=6.6 \mathrm{~Hz}, 18 \mathrm{H}, \mathrm{P}^{t} \mathrm{Bu}\right), 1.30\left(\mathrm{vt}, \mathrm{J}=6.6 \mathrm{~Hz}, 18 \mathrm{H}, \mathrm{P}^{t} \mathrm{Bu}\right) .{ }^{31} \mathrm{P}\left\{{ }^{1} \mathrm{H}\right\} \mathrm{NMR}\left(\mathrm{C}_{6} \mathrm{D}_{6}, 2{ }^{\circ} \mathrm{C}\right)$ (selective hydride-coupled): $65.5 \mathrm{ppm}\left(\mathrm{d}, \mathrm{J}_{\mathrm{PH}}=12.2 \mathrm{~Hz}\right)$.

\section{$(\mathrm{PNP}) \mathrm{Os}(\mathrm{H}) \mathrm{C}=\mathrm{CH}_{2}$}

(PNP)Os $(\mathrm{H})_{3}(10 \mu \mathrm{mol})$ in a J-Young tube was dissolved in $0.5 \mathrm{~mL}$ of $\mathrm{C}_{6} \mathrm{D}_{6}$ and was degassed through 3 freeze-pump-thaw cycles using liquid $\mathrm{N}_{2}$. 1 atm of $\mathrm{HCCH}$ was added to the evacuated head space of the frozen solution in the tube. After 30 minutes of shaking, the volatiles were removed in vacuo. By NMR spectra prior to vacuum drying, formation of $\mathrm{H}_{2} \mathrm{CCH}_{2}$ (5.25 ppm in ${ }^{1} \mathrm{H}$ NMR) was observed, together with complete conversion to (PNP)Os(H)(CCH$)$. ${ }^{1} \mathrm{H}$ NMR $\left(\mathrm{C}_{6} \mathrm{D}_{6}, 25^{\circ} \mathrm{C}\right):-16.13 \mathrm{ppm}\left(\mathrm{t}, \mathrm{J}_{\mathrm{PH}}=13.8 \mathrm{~Hz}\right.$, 1H, OsH), 0.32 (s, 6H, SiMe), 0.35 (s, 6H, SiMe), 0.42 (bs, 2H, C=CH $), 1.24$ (vt, J = 6 
$\left.\mathrm{Hz}, 18 \mathrm{H}, \mathrm{P}^{t} \mathrm{Bu}\right), 1.44\left(\mathrm{vt}, \mathrm{J}=6 \mathrm{~Hz}, 18 \mathrm{H}, \mathrm{P}^{t} \mathrm{Bu}\right) .{ }^{31} \mathrm{P}\left\{{ }^{1} \mathrm{H}\right\} \mathrm{NMR}\left(\mathrm{C}_{6} \mathrm{D}_{6}, 25{ }^{\circ} \mathrm{C}\right): 52.4 \mathrm{ppm}$ (s).

\section{(PNP)OsH(CO) 2}

(PNP)Os $(\mathrm{H})_{3}(10 \mu \mathrm{mol})$ was dissolved in $0.5 \mathrm{~mL}$ of $\mathrm{C}_{6} \mathrm{D}_{6}$ in a J-Young tube and was degassed through 3 freeze-pump-thaw cycles using liquid $\mathrm{N}_{2} .1$ atm of $\mathrm{CO}$ was added into the evacuated head space of the frozen solution in the tube. After 30 minutes, the volatiles were removed in vacuo. By NMR spectra prior to vacuum drying, liberation of $\mathrm{H}_{2}$ (4.46 ppm in ${ }^{1} \mathrm{H}$ NMR) was detected. ${ }^{1} \mathrm{H}$ NMR $\left(\mathrm{C}_{6} \mathrm{D}_{6}, 25^{\circ} \mathrm{C}\right)$ : - 4.83ppm (t, $\mathrm{J}_{\mathrm{HP}}=$ $20 \mathrm{~Hz}, 1 \mathrm{H}, \mathrm{OsH}$ ), 0.31 (s, 6H, SiMe), 0.38 (s, 6H, SiMe), 1.25 (vt, J = 6.4 Hz, 18H, ${ }^{t} B u$ ), 1.26 (vt, J = 6.4 Hz, 18H, $\left.\mathrm{P}^{t} \mathrm{Bu}\right) .{ }^{31} \mathrm{P}\left\{{ }^{1} \mathrm{H}\right\} \operatorname{NMR}\left(\mathrm{C}_{6} \mathrm{D}_{6}, 25^{\circ} \mathrm{C}\right): 52.9 \mathrm{ppm}(\mathrm{s})$, selective coupling of Os- $H\left(\mathrm{~d}, \mathrm{~J}_{\mathrm{HP}}=12.5 \mathrm{~Hz}\right)$. IR (pentane solution): $v(\mathrm{C} \equiv \mathrm{O}) 1898 \mathrm{~cm}^{-1}, 1963 \mathrm{~cm}^{-1}$.

\section{Isotope labeling experiments:}

\section{$(\mathrm{PNP}) \mathrm{Os}(\mathrm{D})_{3}$}

(PNP)Os $(\mathrm{H})_{3}(10 \mu \mathrm{mol})$ was dissolved in $0.5 \mathrm{~mL}$ of $\mathrm{C}_{6} \mathrm{D}_{6}$ in a J-Young tube and was degassed through 3 freeze-pump-thaw cycles using liquid $\mathrm{N}_{2}$. 1 atm of $\mathrm{D}_{2}$ was added to the evacuated head space of the frozen solution in the tube. After 1day of agitation, the volatiles were removed in vacuo. ${ }^{1} \mathrm{H}$ NMR $\left(\mathrm{C}_{6} \mathrm{D}_{6}, 25^{\circ} \mathrm{C}\right)$ : $0.42 \mathrm{ppm}(\mathrm{s}, 12 \mathrm{H}, \mathrm{SiMe})$, 0.94 (vt, J = 4.6 Hz, 4H, SiCH $), 1.19$ (vt, J = 6.6 Hz, 36H, P $\left.{ }^{t} \mathrm{Bu}\right) .{ }^{2} \mathrm{H}$ NMR $\left(\mathrm{C}_{6} \mathrm{D}_{6}, 25\right.$ $\left.{ }^{\circ} \mathrm{C}\right)$ : -16.38 ppm (bs, 3D, Os $\left.D_{3}\right) .{ }^{31} \mathrm{P}\left\{{ }^{1} \mathrm{H}\right\}$ NMR $\left(\mathrm{C}_{6} \mathrm{D}_{6}, 25{ }^{\circ} \mathrm{C}\right): 70 \mathrm{ppm}(\mathrm{s})$.

\section{(PNP)Os $\left({ }^{13} \mathrm{CO}\right)\left(\mathrm{CH}_{2} \mathrm{Ar}\right)$}

(PNP)Os $(\mathrm{H})_{2}(\mathrm{CAr})(10 \mu \mathrm{mol})$ in a J-Young tube was dissolved in $0.5 \mathrm{~mL}$ of $\mathrm{C}_{6} \mathrm{D}_{6}$ and was degassed through 3 freeze-pump-thaw cycles using liquid $\mathrm{N}_{2} .0 .5 \mathrm{~atm}$ of ${ }^{13} \mathrm{CO}$ was added to the evacuated head space of the frozen solution in the tube and the solution was melted and shaken. Immediate color changed was observed from redish brown to 
green. After 30 minutes, the reaction tube was placed into a $65^{\circ} \mathrm{C}$ bath for 4 hours. A color change was observed from green to brown. The volatiles were removed in vacuo. ${ }^{1} \mathrm{H}$ NMR $\left(\mathrm{C}_{6} \mathrm{D}_{6}, 25^{\circ} \mathrm{C}\right): 0.40 \mathrm{ppm}(\mathrm{s}, 6 \mathrm{H}, \mathrm{SiMe}), 0.47$ (s, 6H, SiMe), 1.11 (vt, J = 6.4 Hz, 18H, $\left.\mathrm{P}^{t} B u\right), 1.19\left(\mathrm{vt}, \mathrm{J}=6.4 \mathrm{~Hz}, 18 \mathrm{H}, \mathrm{P}^{t} B u\right), 2.73\left(\mathrm{sep}, \mathrm{J}_{\mathrm{HH}}=6.8 \mathrm{~Hz} 1 \mathrm{H}, \mathrm{CHMe}\right.$ ), $4.76(\mathrm{t}$, $\left.\mathrm{J}_{\mathrm{HP}}=4.8 \mathrm{~Hz}, 2 \mathrm{H}, \mathrm{CH}_{2} \mathrm{Ar}\right), 7.06\left(\mathrm{~d}, \mathrm{~J}_{\mathrm{HH}}=8.4 \mathrm{~Hz}, 2 \mathrm{H}, A r\right), 7.66\left(\mathrm{~d}, \mathrm{~J}_{\mathrm{HH}}=8.0 \mathrm{~Hz}, 2 \mathrm{H}, A r\right)$. ${ }^{31} \mathrm{P}\left\{{ }^{1} \mathrm{H}\right\} \operatorname{NMR}\left(\mathrm{C}_{6} \mathrm{D}_{6}, 25{ }^{\circ} \mathrm{C}\right): 38.8 \mathrm{ppm}\left(\mathrm{d}, \mathrm{J}_{\mathrm{CP}}=10 \mathrm{~Hz}, P^{\mathrm{t}} \mathrm{Bu}_{2}\right) \cdot{ }^{13} \mathrm{C}\left\{{ }^{1} \mathrm{H}\right\} \mathrm{NMR}\left(\mathrm{C}_{6} \mathrm{D}_{6}, 25\right.$ $\left.{ }^{\circ} \mathrm{C}\right): 188.7 \mathrm{ppm}\left(\mathrm{t}, \mathrm{J}_{\mathrm{CP}}=9.3 \mathrm{~Hz}, \mathrm{OsCO}\right)$. IR (pentane solution): $v(\mathrm{C} \equiv \mathrm{O}) 1820 \mathrm{~cm}^{-1}$.

\section{$(\mathrm{PNP}) \mathrm{Os}(\mathrm{H})_{3}$ and DCCD}

(PNP)Os $(\mathrm{H})_{3}(10 \mu \mathrm{mol})$ was dissolved in $0.5 \mathrm{~mL}$ of $\mathrm{C}_{6} \mathrm{D}_{6}$ in a J-Young tube and was degassed through 3 freeze-pump-thaw cycles using liquid $\mathrm{N}_{2} .0 .5 \mathrm{~atm}$ of DCCD was added into the evacuated head space of the frozen solution in the tube. After 30 minutes, the volatiles were removed in vacuo. ${ }^{1} \mathrm{H}$ NMR $\left(\mathrm{C}_{6} \mathrm{D}_{6}, 25^{\circ} \mathrm{C}\right)$ : $-16.13 \mathrm{ppm}(\mathrm{m}, 1 \mathrm{H}, \mathrm{Os} H)$, 0.33 (s, 6H, SiMe), 0.36 (s, 6H, SiMe), 1.24 (vt, J = 6 Hz, 18H, P ${ }^{t} B u$ ), 1.44 (vt, J = $6 \mathrm{~Hz}$, $\left.18 \mathrm{H}, \mathrm{P}^{t} \mathrm{Bu}\right) .{ }^{2} \mathrm{H}$ NMR $\left(\mathrm{C}_{6} \mathrm{D}_{6}, 25{ }^{\circ} \mathrm{C}\right):-15.95 \mathrm{ppm}$ (bs, OsD), 0.48 (bs, $\left.\mathrm{C}=\mathrm{CD}_{2}\right) .{ }^{31} \mathrm{P}\left\{{ }^{1} \mathrm{H}\right\}$ $\operatorname{NMR}\left(\mathrm{C}_{6} \mathrm{D}_{6}, 25^{\circ} \mathrm{C}\right): 52.5 \mathrm{ppm}(\mathrm{s})$.

\section{(PNP)Os(H) $)_{3}$ and $\mathrm{H}^{13} \mathrm{C}^{13} \mathrm{CH}$}

(PNP)Os $(\mathrm{H})_{3}(10 \mu \mathrm{mol})$ in a J-Young tube was dissolved in $0.5 \mathrm{~mL}$ of $\mathrm{C}_{6} \mathrm{D}_{6}$ and was degassed through 3 freeze-pump-thaw cycles using liquid $\mathrm{N}_{2} .0 .5 \mathrm{~atm}$ of $\mathrm{H}^{13} \mathrm{C}^{13} \mathrm{CH}$ was added into the evacuated head space of the frozen solution in the tube. After agitation for 10 minutes, NMR spectra were collected. $\mathrm{H}_{2}{ }^{13} \mathrm{C}^{13} \mathrm{CH}_{2}$ was observed in ${ }^{1} \mathrm{H}$ NMR spectra $\left(5.23 \mathrm{ppm}, \mathrm{J}_{\mathrm{CH}}=153.9 \mathrm{~Hz}\right) .{ }^{1} \mathrm{H}$ NMR $\left(\mathrm{C}_{6} \mathrm{D}_{6}, 25{ }^{\circ} \mathrm{C}\right):-16.13 \mathrm{ppm}\left(\mathrm{dt}, \mathrm{J}_{\mathrm{CH}}=\right.$ $\left.6.9 \mathrm{~Hz}, \mathrm{~J}_{\mathrm{PH}}=13.8 \mathrm{~Hz}, 1 \mathrm{H}, \mathrm{Os} H\right), 0.32(\mathrm{~s}, 6 \mathrm{H}, \mathrm{SiMe}), 0.35$ (s, 6H, SiMe), $0.42\left(\mathrm{dm}, \mathrm{J}_{\mathrm{CH}}=\right.$ $\left.160 \mathrm{~Hz}, 2 \mathrm{H},=\mathrm{CH}_{2}\right), 1.24\left(\mathrm{vt}, \mathrm{J}=6 \mathrm{~Hz}, 18 \mathrm{H}, \mathrm{P}^{t} \mathrm{Bu}\right), 1.44\left(\mathrm{vt}, \mathrm{J}=6 \mathrm{~Hz}, 18 \mathrm{H}, \mathrm{P}^{t} \mathrm{Bu}\right)$.

${ }^{31} \mathrm{P}\left\{{ }^{1} \mathrm{H}\right\} \operatorname{NMR}\left(\mathrm{C}_{6} \mathrm{D}_{6}, 25{ }^{\circ} \mathrm{C}\right): 52.4 \mathrm{ppm}\left(\mathrm{dd}, \mathrm{J}_{\mathrm{CP}}=10 \mathrm{~Hz}, \mathrm{~J}_{\mathrm{CP}}=3.6 \mathrm{~Hz}\right) .{ }^{13} \mathrm{C}\left\{{ }^{1} \mathrm{H}\right\} \mathrm{NMR}$ $\left(\mathrm{C}_{6} \mathrm{D}_{6}, 25^{\circ} \mathrm{C}\right): 285.6 \mathrm{ppm}\left(\mathrm{td}, \mathrm{J}_{\mathrm{CP}}=9.8 \mathrm{~Hz}, \mathrm{~J}_{\mathrm{CC}}=61 \mathrm{~Hz}, \mathrm{OsC}\right), 90.6 \mathrm{ppm}\left(\mathrm{dd}, \mathrm{J}_{\mathrm{CC}}=61 \mathrm{~Hz}\right.$, $\mathrm{J}_{\mathrm{cp}}=3.4 \mathrm{~Hz}, \mathrm{Os}=\mathrm{C}=\mathrm{CH}_{2}$ ). 


\section{(PNP)OsH $\left({ }^{13} \mathrm{CO}\right)$}

(PNP)Os $\left({ }^{13} \mathrm{CO}\right)\left(\mathrm{CH}_{2} \mathrm{Ar}\right)(10 \mu \mathrm{mol})$ in a J-Young tube was dissolved in $0.5 \mathrm{~mL}$ of $\mathrm{C}_{6} \mathrm{D}_{6}$ and was degassed through 3 freeze-pump-thaw cycles using liquid $\mathrm{N}_{2}$. 1 atm of $\mathrm{H}_{2}$ was added to the evacuated head space of the frozen solution in the tube and the solution was melted and shaken. Immediate color changed was observed from brown to pale yellow. After 30 minutes, the volatiles were removed in vacuo. ${ }^{1} \mathrm{H}$ NMR $\left(\mathrm{C}_{6} \mathrm{D}_{6}\right.$, $\left.25^{\circ} \mathrm{C}\right):-29.46 \mathrm{ppm}\left(\mathrm{dt}, \mathrm{J}_{\mathrm{CH}}=10.8 \mathrm{~Hz}, \mathrm{~J}_{\mathrm{PH}}=13.2 \mathrm{~Hz}, 1 \mathrm{H}, \mathrm{OsH}\right), 0.32(\mathrm{~s}, 6 \mathrm{H}, \mathrm{SiMe}), 0.33$ (s, 6H, SiMe), 1.23 (vt, J = 6.6 Hz, 18H, ${ }^{t} B u$ ), 1.30 (vt, J = 6.6 Hz, 18H, ${ }^{t} B u$ ).

${ }^{31} \mathrm{P}\{$ selectively hydride-coupled $\} \operatorname{NMR}\left(\mathrm{C}_{6} \mathrm{D}_{6}, 25^{\circ} \mathrm{C}\right): 65.5 \mathrm{ppm}\left(\mathrm{dd}, \mathrm{J}_{\mathrm{CP}}=9.1 \mathrm{~Hz}, \mathrm{~J}_{\mathrm{PH}}=\right.$ $12.2 \mathrm{~Hz})$.

\section{Reaction of (PNP)OsH $\left({ }^{13} \mathrm{CO}\right)$ with $\mathrm{CO}$}

(PNP)OsH $\left({ }^{13} \mathrm{CO}\right)(10 \mu \mathrm{mol})$ was dissolved in $0.5 \mathrm{~mL}$ of $\mathrm{C}_{6} \mathrm{D}_{6}$ in a $\mathrm{J}$-Young tube and was degassed through 3 freeze-pump-thaw cycles using liquid $\mathrm{N}_{2}$. 1 atm of isotopically normal $\mathrm{CO}$ was added to the evacuated head space of the frozen solution in the tube. After agitation for 30 minutes, the volatiles were removed in vacuo. ${ }^{1} \mathrm{H}$ NMR $\left(\mathrm{C}_{6} \mathrm{D}_{6}, 25^{\circ} \mathrm{C}\right):-4.82 \mathrm{ppm}\left(\mathrm{dt}, \mathrm{J}_{\mathrm{CH}}=6 \mathrm{~Hz}, \mathrm{~J}_{\mathrm{PH}}=19.8 \mathrm{~Hz}, 1 \mathrm{H}, \mathrm{OsH}\right), 0.31(\mathrm{~s}, 6 \mathrm{H}, \mathrm{SiMe})$, 0.38 (s, 6H, SiMe), 1.25 (vt, J = 6.6 Hz, 18H, $\mathrm{P}^{t} \mathrm{Bu}$ ), 1.27 (vt, J = 6.6 Hz, 18H, $\mathrm{P}^{t} \mathrm{Bu}$ ). ${ }^{13} \mathrm{C}\left\{{ }^{1} \mathrm{H}\right\} \operatorname{NMR}\left(\mathrm{C}_{6} \mathrm{D}_{6}, 25{ }^{\circ} \mathrm{C}\right): 187.4 \mathrm{ppm}\left(\mathrm{dt}, \mathrm{J}_{\mathrm{CC}}=103.1 \mathrm{~Hz}, \mathrm{~J}_{\mathrm{CP}}=8.6 \mathrm{~Hz}, \mathrm{CO}\right), 183.5(\mathrm{~d}$, $\left.\mathrm{J}_{\mathrm{CP}}=8.6 \mathrm{~Hz},{ }^{13} \mathrm{CO}\right) .{ }^{31} \mathrm{P}\left\{{ }^{1} \mathrm{H}\right\} \operatorname{NMR}\left(\mathrm{C}_{6} \mathrm{D}_{6}, 25{ }^{\circ} \mathrm{C}\right): 53.1 \mathrm{ppm}\left(\mathrm{d}, \mathrm{J}_{\mathrm{CP}}=8.2 \mathrm{~Hz}\right)$. 


\section{$(\mathrm{PNP}) \mathrm{Os}(\mathrm{H})_{2}(=\mathrm{CAr})$}

${ }^{1} \mathrm{H}$ NMR (300 MHz, $\left.\mathrm{C}_{6} \mathrm{D}_{6}\right)$

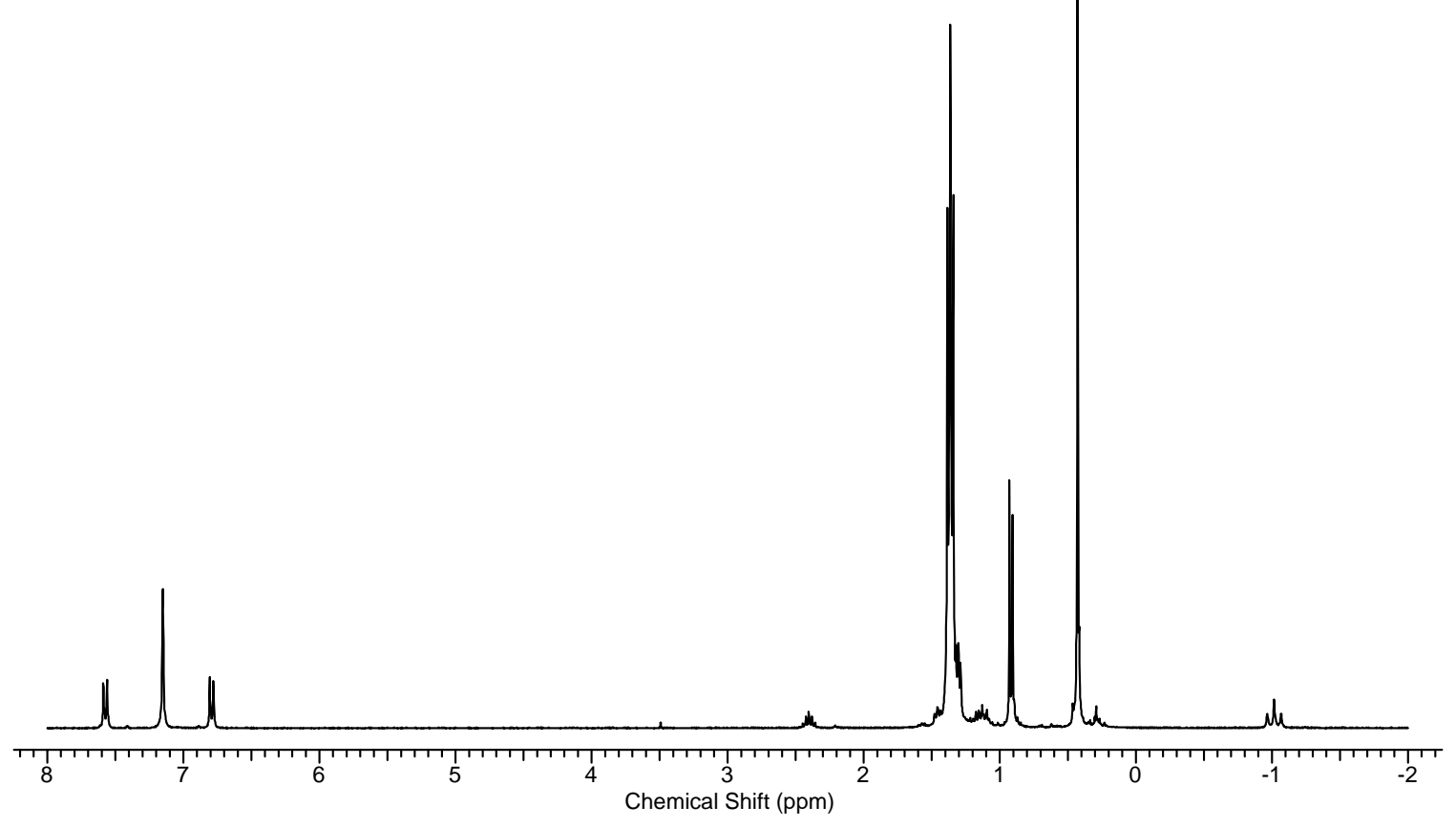

${ }^{31} \mathrm{P}\left\{{ }^{1} \mathrm{H}\right\}$ NMR (121 MHz, $\left.\mathrm{C}_{6} \mathrm{D}_{6}\right)$

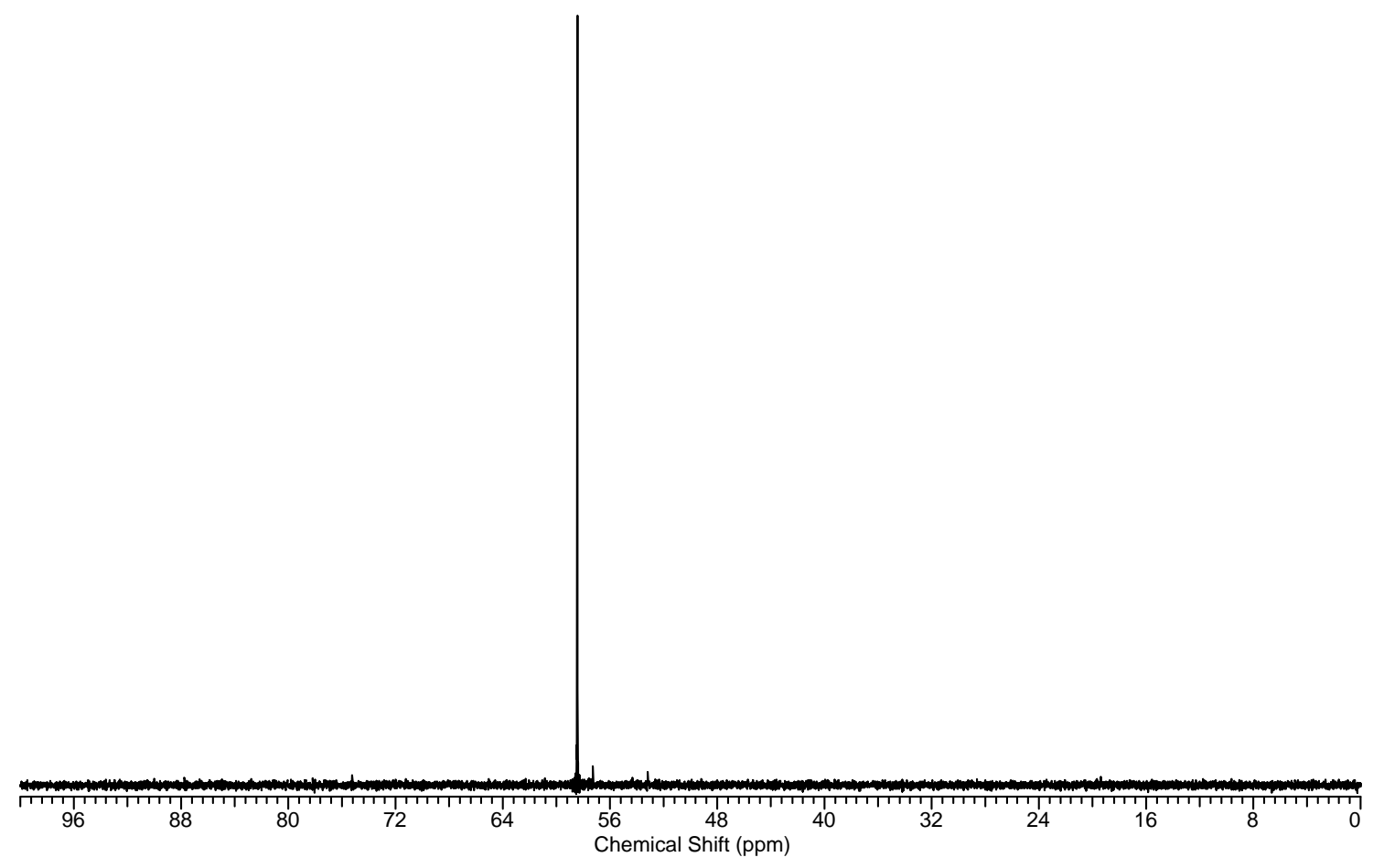


(PNP)OsH 3

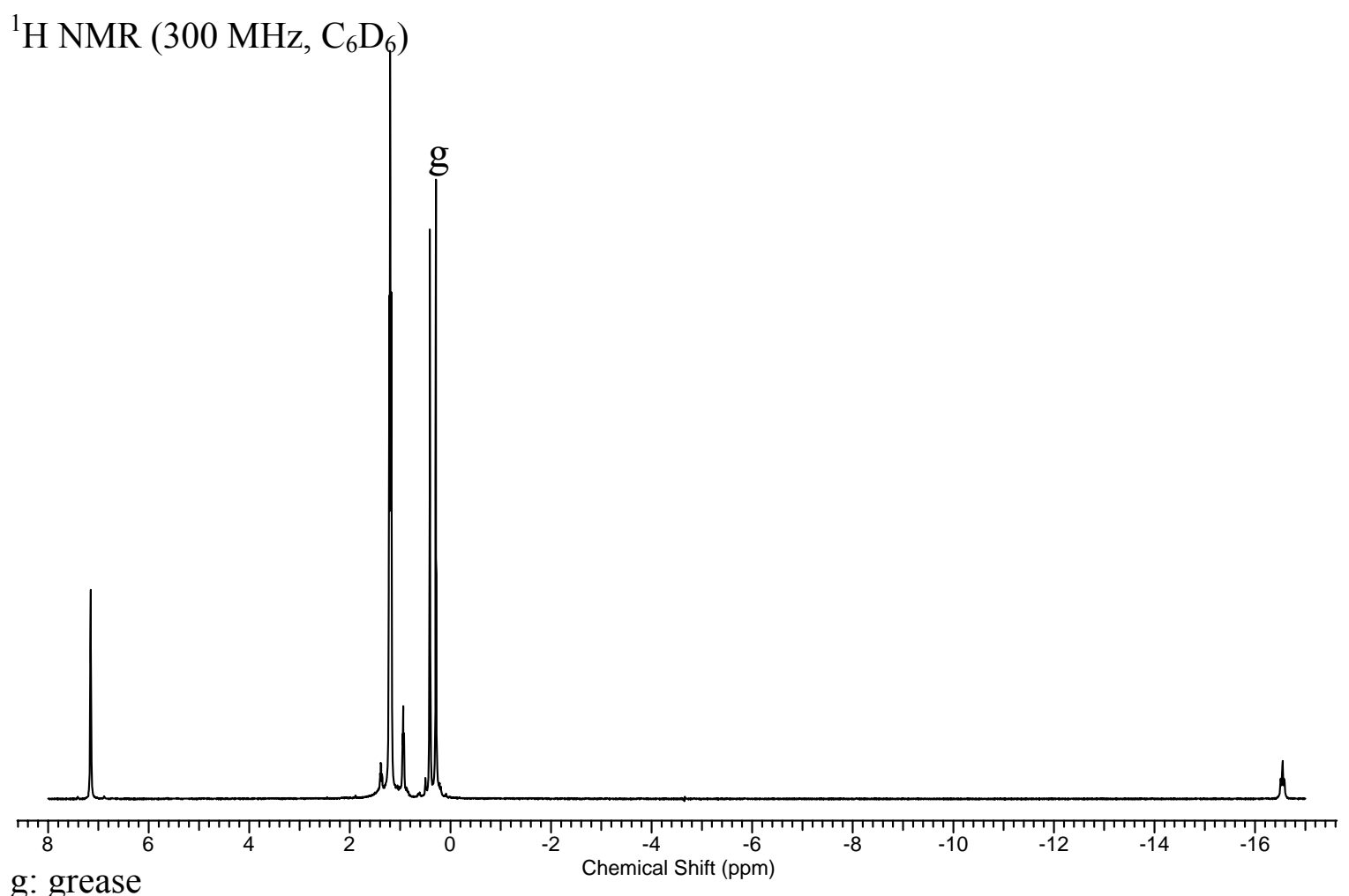

g: grease

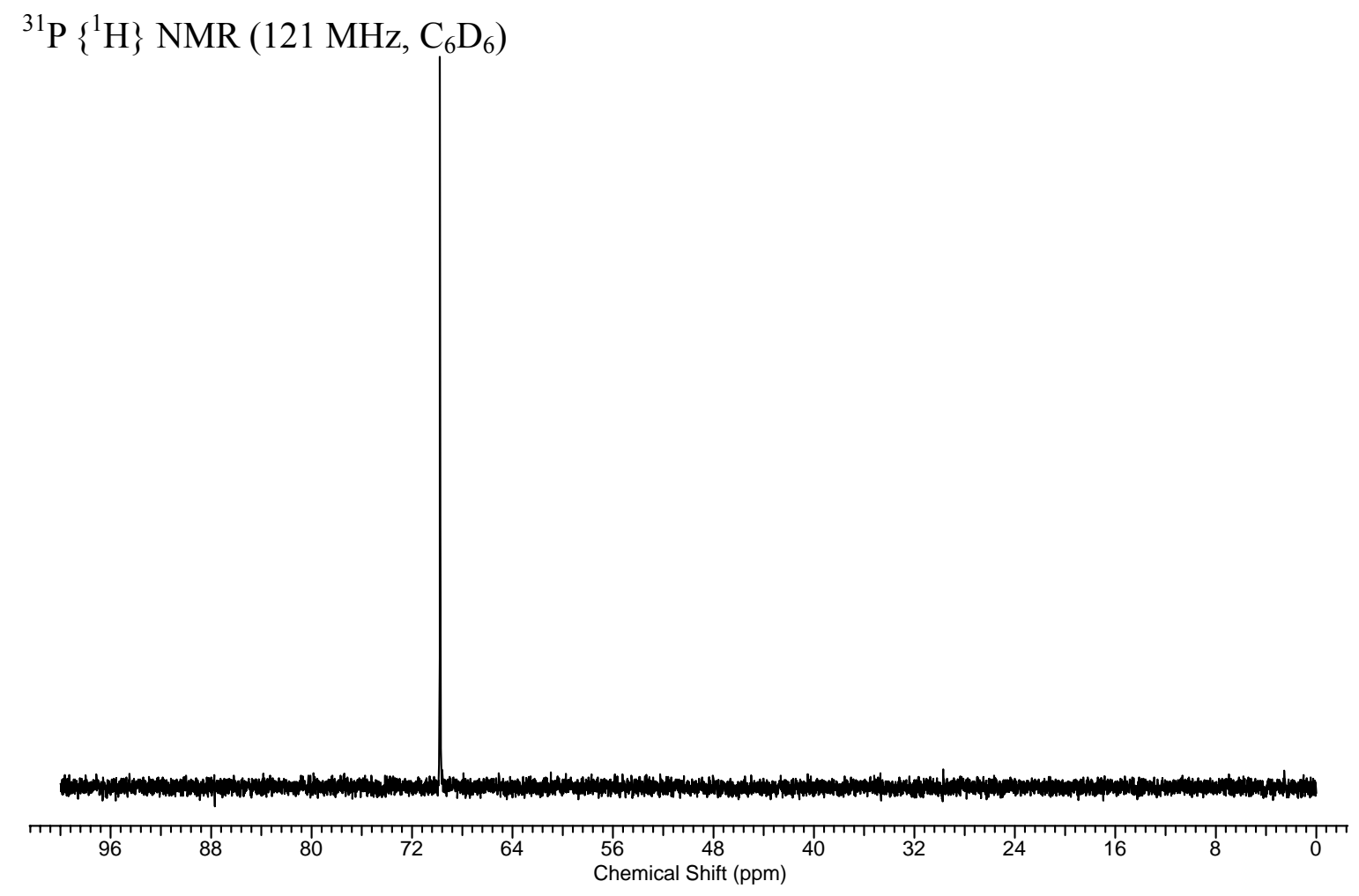




\section{(PNP)OsH(CO)(=C(H)Ar)}

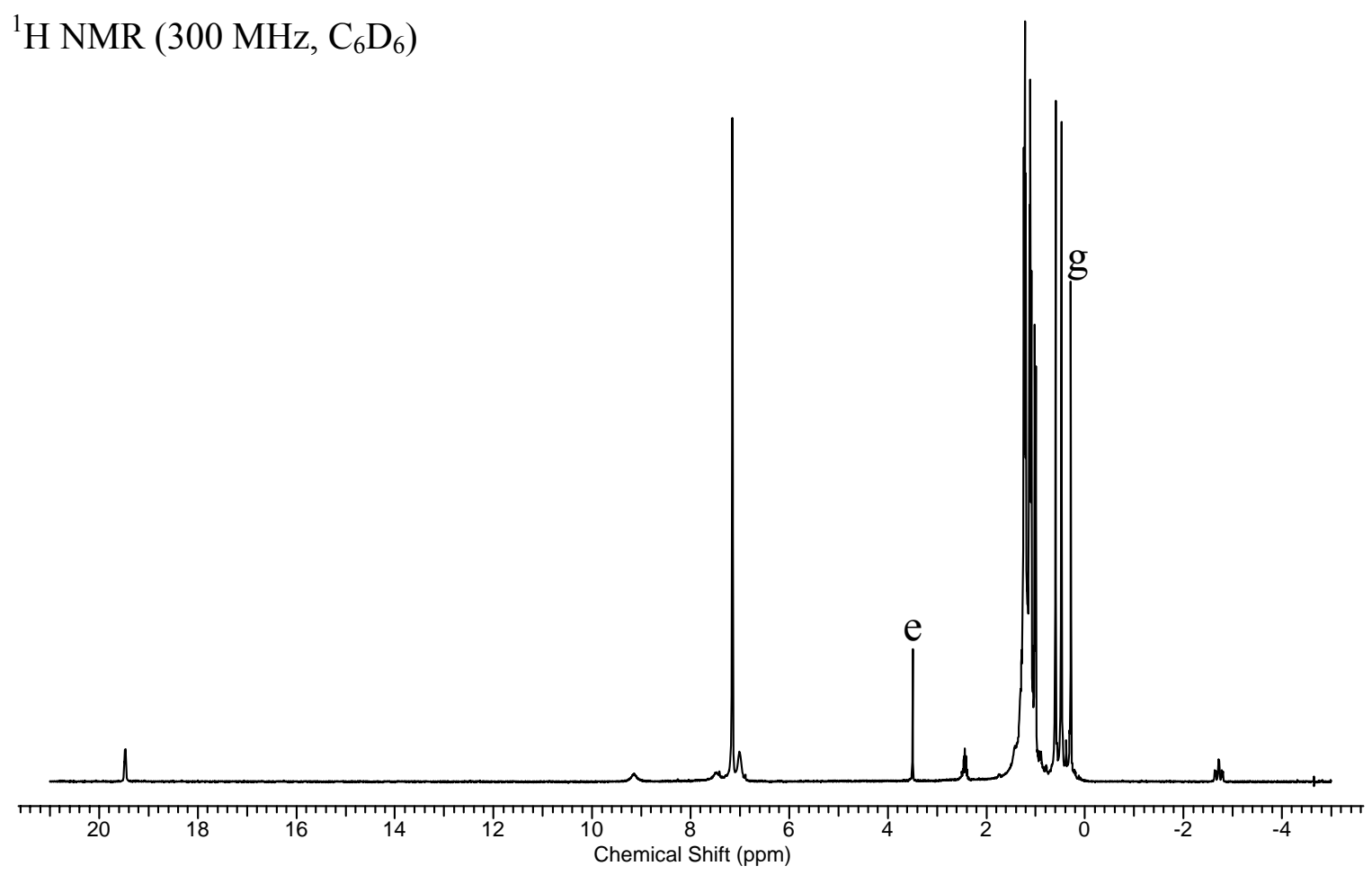

e: 12 -crown-4 ether, g: grease

${ }^{31} \mathrm{P}\left\{{ }^{1} \mathrm{H}\right\}$ NMR (121 MHz, $\left.\mathrm{C}_{6} \mathrm{D}_{6}\right)$

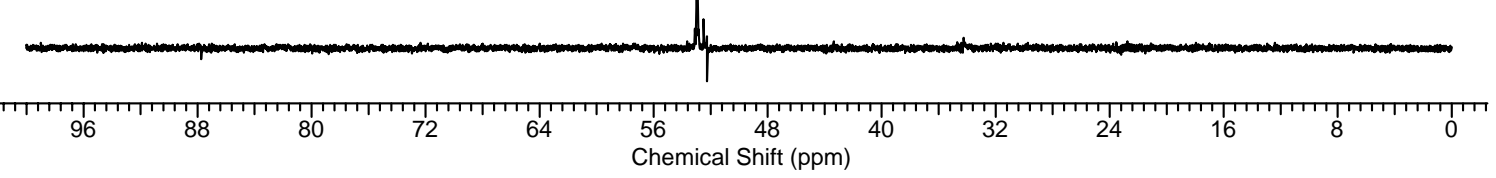




\section{$(\mathrm{PNP}) \mathrm{Os}(\mathrm{CO})\left(\mathrm{CH}_{2} \mathrm{Ar}\right)$}

${ }^{1} \mathrm{H}$ NMR (300 MHz, $\mathrm{C}_{6} \mathrm{D}_{6}$ )

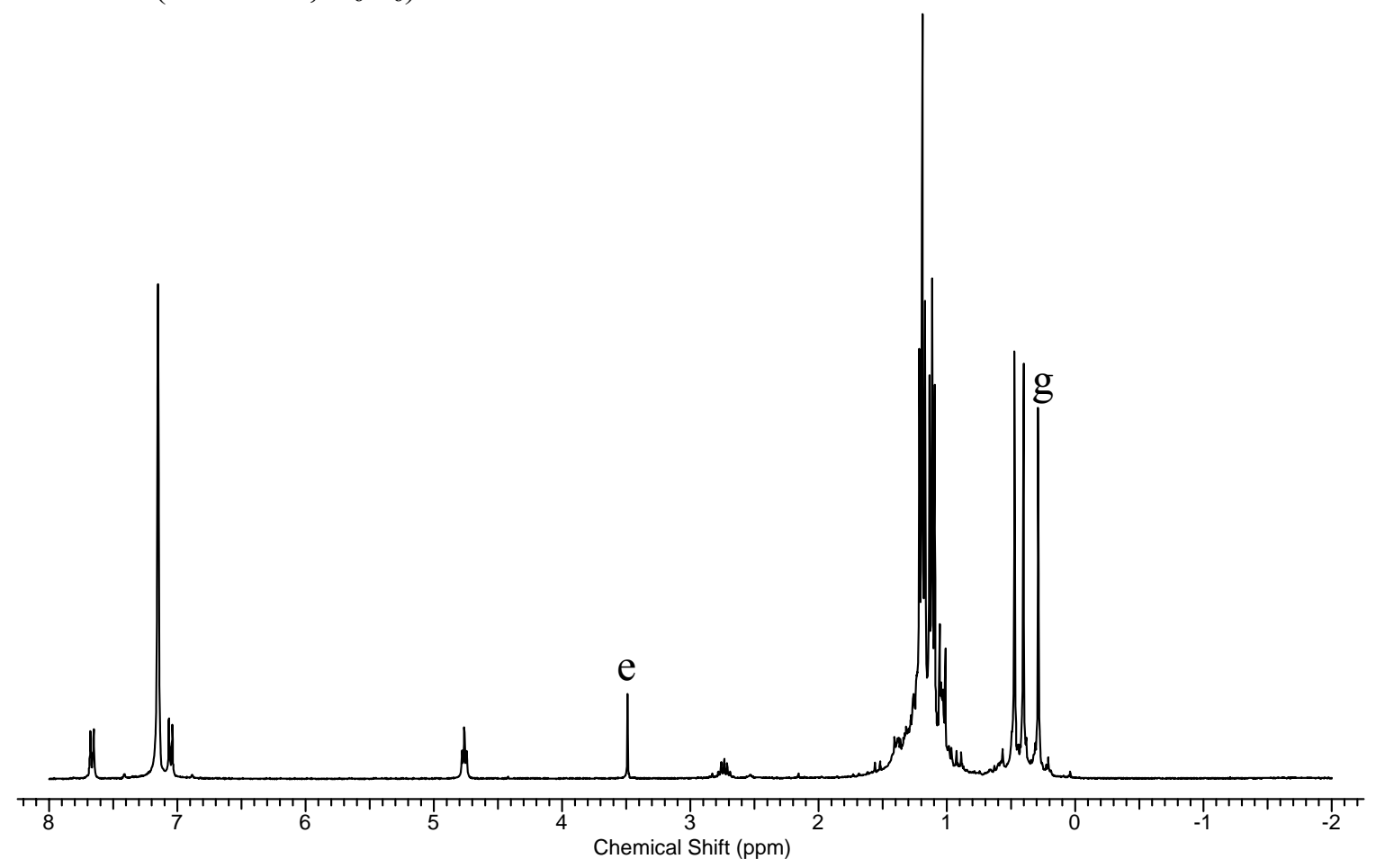

e: 12-crown-4 ether, g: grease

${ }^{31} \mathrm{P}\left\{{ }^{1} \mathrm{H}\right\}$ NMR (121 MHz, $\left.\mathrm{C}_{6} \mathrm{D}_{6}\right)$

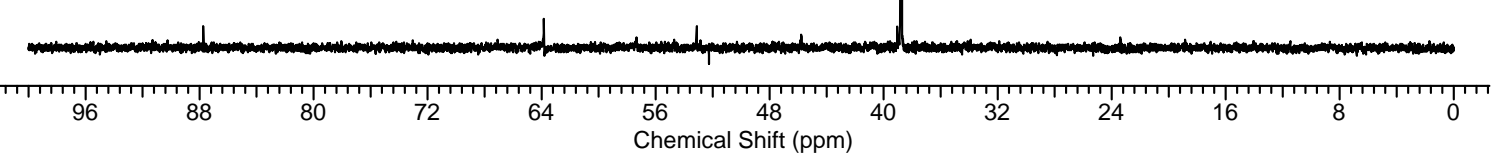


(PNP)OsH(CO)

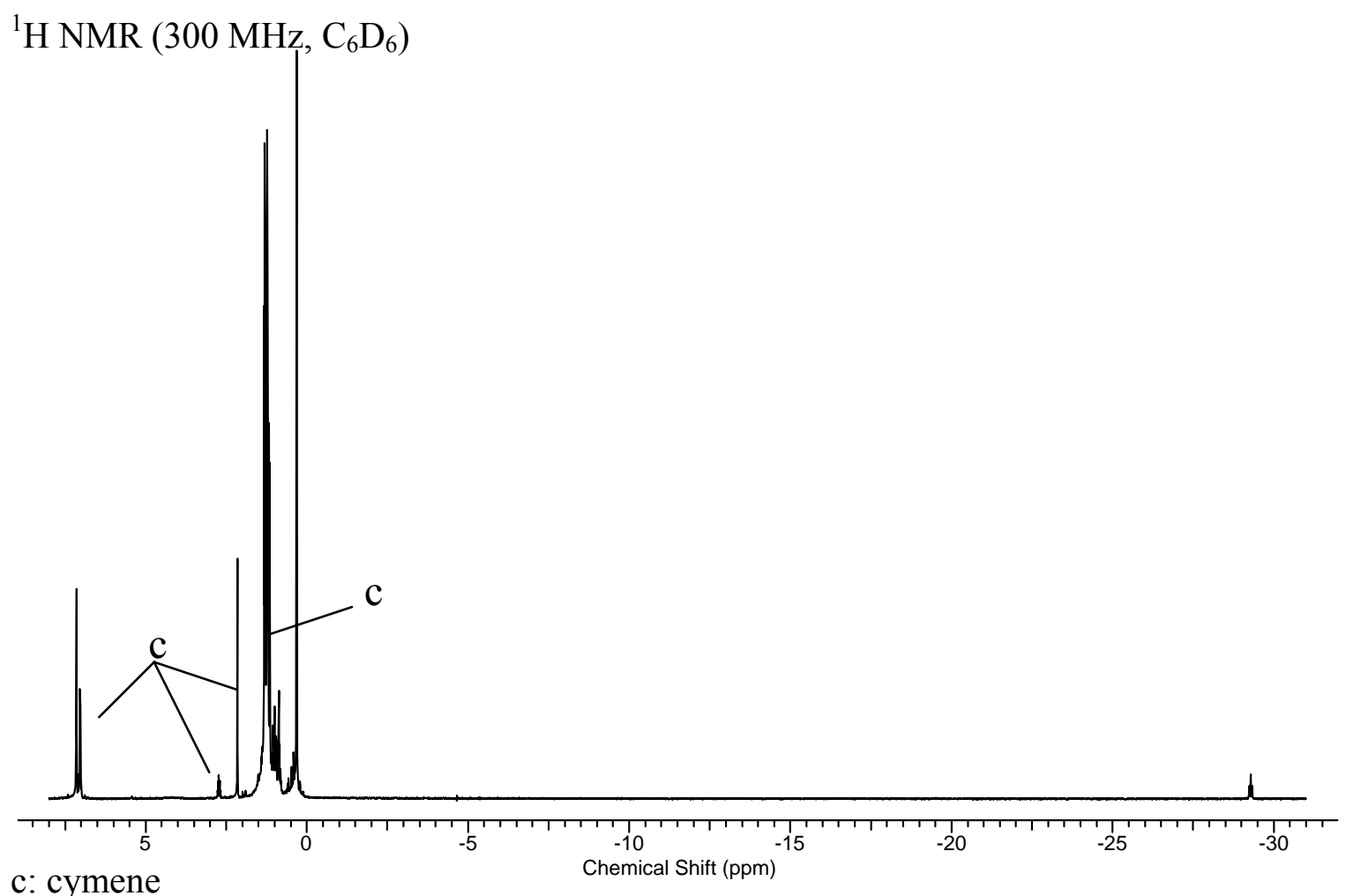

c: cymene

${ }^{31} \mathrm{P}\left\{{ }^{1} \mathrm{H}\right\} \mathrm{NMR}\left(121 \mathrm{MHz}, \mathrm{C}_{6} \mathrm{D}_{6}\right)$

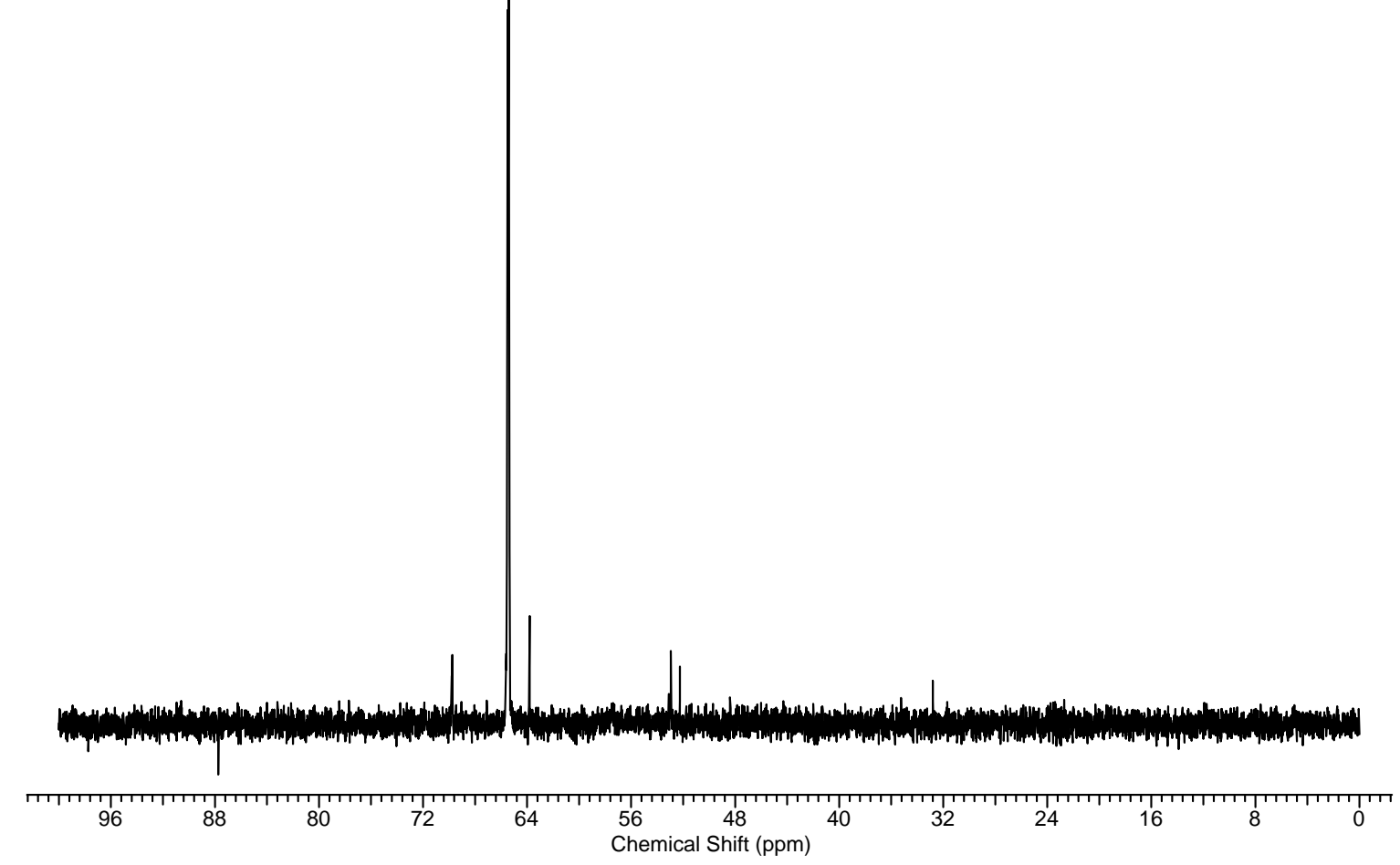


$(\mathrm{PNP}) \mathrm{OsH}\left(=\mathrm{C}=\mathrm{CH}_{2}\right)$

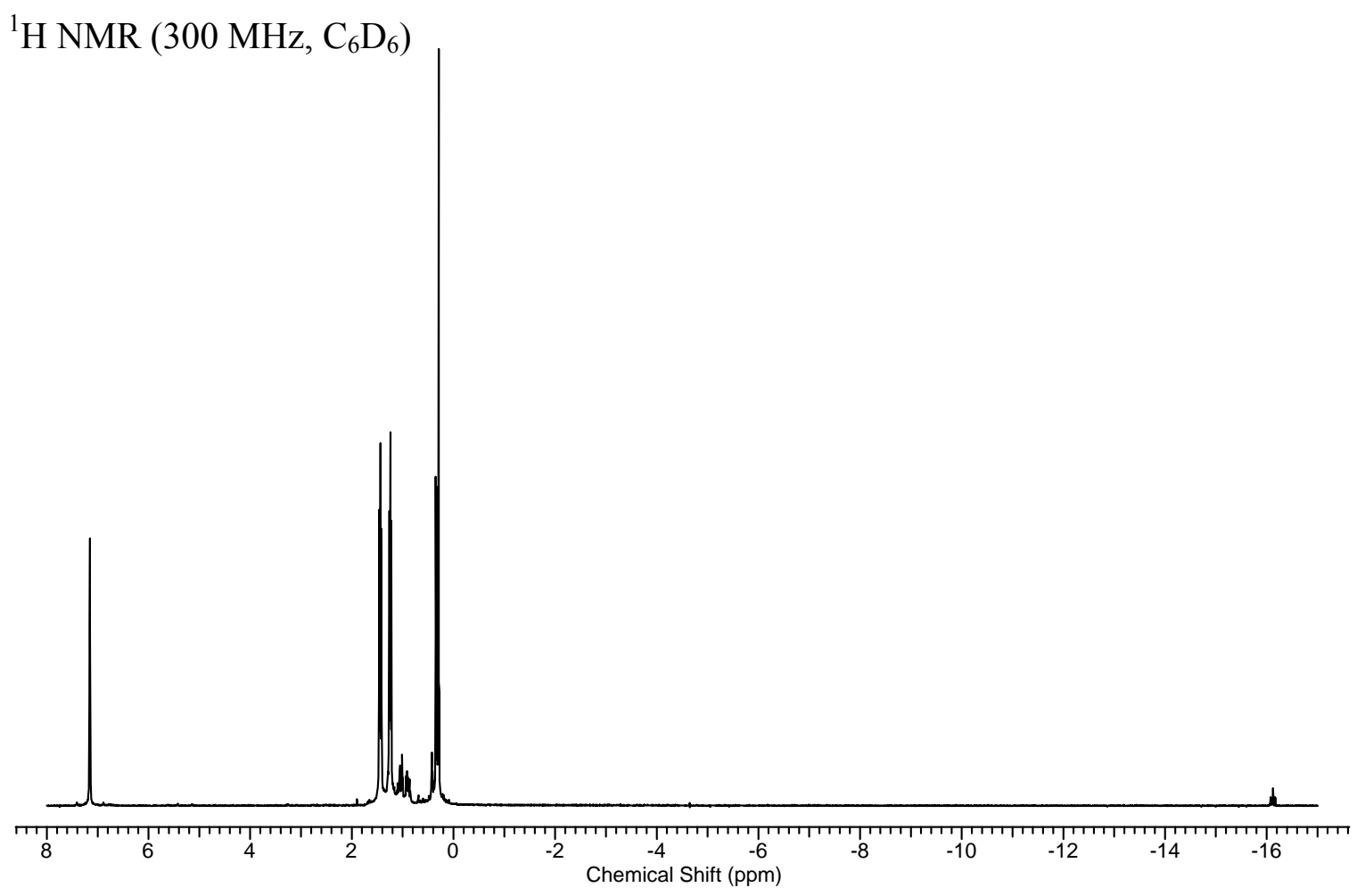

${ }^{31} \mathrm{P}\left\{{ }^{1} \mathrm{H}\right\}$ NMR $\left(121 \mathrm{MHz}, \mathrm{C}_{6} \mathrm{D}_{6}\right)$

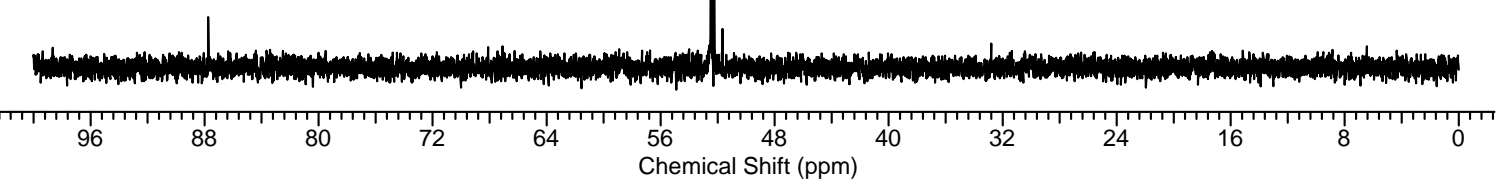




\section{(PNP)OsH(CO)}

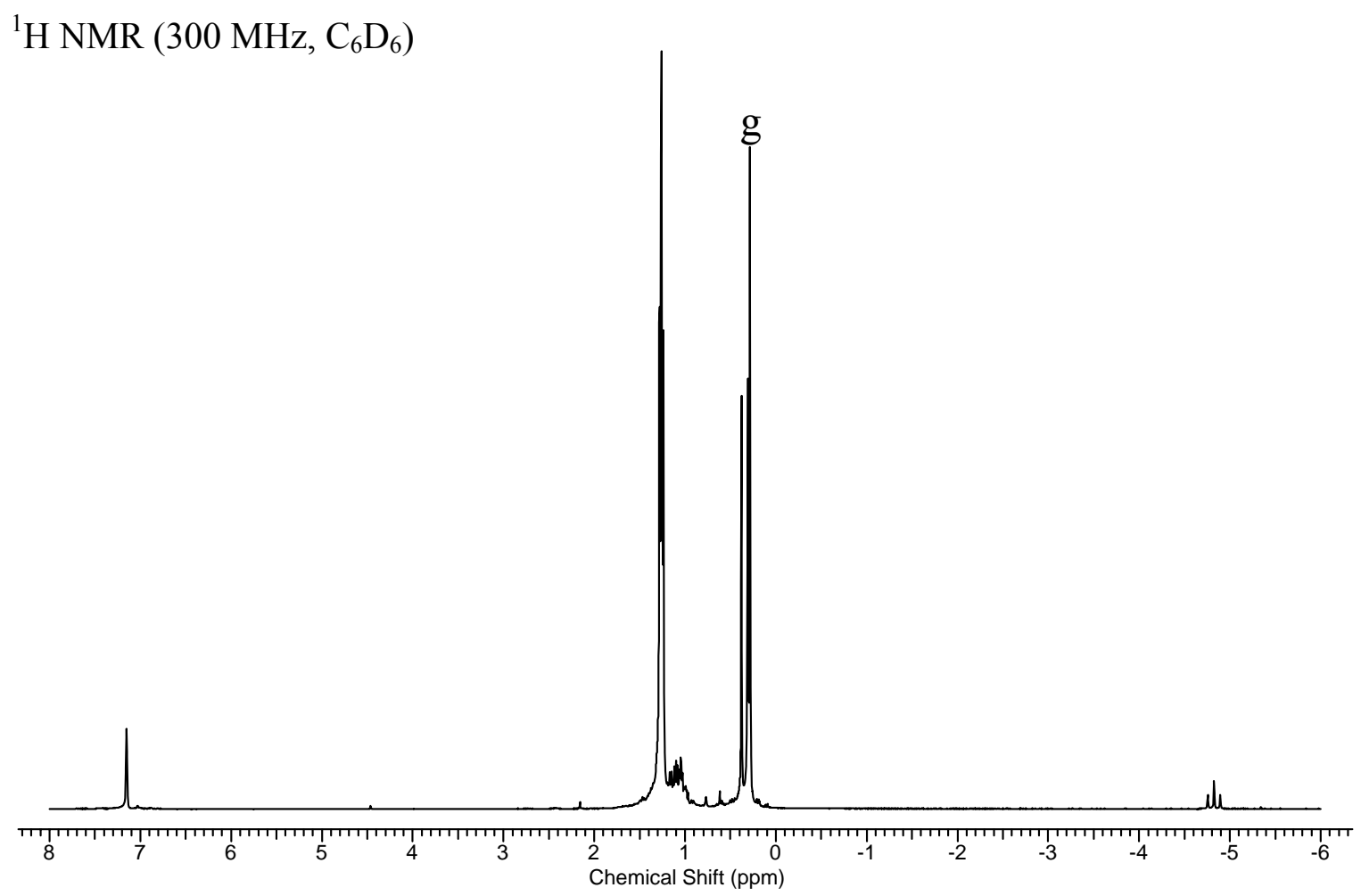

g: grease

${ }^{31} \mathrm{P}\left\{{ }^{1} \mathrm{H}\right\}$ NMR (121 MHz, $\left.\mathrm{C}_{6} \mathrm{D}_{6}\right)$

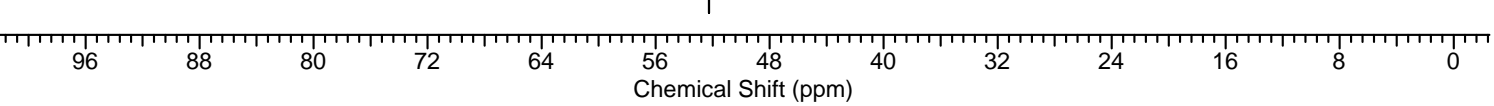

